\title{
The ATLAS Hadronic Tile Calorimeter: From Construction Toward Physics
}

P. Adragna, C. Alexa, K. Anderson, A. Antonaki, V. Batusov, P. Bednar, S. Binet, C. Biscarat, G. Blanchot, A. Bogush, C. Bohm, V. Boldea, M. Bosman, C. Bromberg, J. Budagov, L. Caloba, D. Calvet, J. Carvalho, J. Castelo, M. V. Castillo, M. Cavalli Sforza, V. Cavasinni, A. S. Cerqueira, R. Chadelas, D. Costanzo, F. Cogswell, S. Constantinescu, M. Crouau, C. Cuenca, D. O. Damazio, F. Daudon, M. David, T. Davidek, K. De, T. Del Prete, B. Di Girolamo, S. Dita, J. Dolejsi, Z. Dolezal, A. Dotti, R. Downing, I. Efthymiopoulos, D. Errede, S. Errede, A. Farbin, D. Fassouliotis, I. Fedorko, A. Fenyuk, C. Ferdi, A. Ferrer, V. Flaminio, E. Fullana, V. Garde, V. Giakoumopoulou, O. Gildemeister, V. Gilewsky, V. Giangiobbe, N. Giokaris, A. Gomes, V. Gonzalez, V. Grabsky, P. Grenier, P. Gris, V. Guarino, C. Guicheney, A. Gupta, H. Hakobyan, M. Haney, A. Henriques, E. Higon, S. Holmgren, M. Hurwitz, J. Huston, C. Iglesias, K. Jon And, T. Junk, A. Karyukhin, J. Khubua, J. Klereborn, I. Korolkov, P. Krivkova, Y. Kulchitsky, Yu. Kurochkin, P. Kuzhir, D. Lambert, T. Le Compte, R. Lefevre, R. Leitner, M. Lembesi, J. Li, M. Liablin, M. Lokajicek, Y. Lomakin, J. M. Lopez Amengual, A. Lupi, C. Maidantchik, A. Maio, S. Maliukov, A. Manousakis, C. Marques, F. Marroquim, F. Martin, E. Mazzoni, G. Montarou, F. Merritt, A. Miagkov, R. Miller, I. Minashvili, L. Miralles, S. Nemecek, M. Nessi, L. Nodulman, O. Norniella, A. Onofre, M. Oreglia, D. Pantea, D. Pallin, J. Pilcher, J. Pina, J. Pinhão, F. Podlyski, X. Portell, J. Poveda, L. E. Price, L. Pribyl, J. Proudfoot, M. Ramstedt, G. Reinmuth, R. Richards, C. Roda, V. Romanov, P. Rosnet, P. Roy, V. Rumiantsau, N. Russakovich, O. Saltò, B. Salvachua, E. Sanchis, H. Sanders, C. Santoni, J. Santos, J. G. Saraiva, F. Sarri, I. Satsunkevitch, L.-P. Says, G. Schlager, J. Schlereth, J. M. Seixas, B. Selldèn, P. Shevtsov, M. Shochet, P. Da Silva, J. Silva, V. Simaitis, A. Sissakian, A. Solodkov, O. Solovianov, M. Sosebee, F. Spanó, R. Stanek, E. Starchenko, P. Starovoitov, M. Suk, I. Sykora, F. Tang, P. Tas, R. Teuscher, S. Tokar, N. Topilin, J. Torres, V. Tsulaia, D. Underwood, G. Usai, S. Valkar, J. A. Valls, A. Vartapetian, F. Vazeille, I. Vichou, V. Vinogradov, I. Vivarelli, M. Volpi, A. White, A. Zaitsev, A. Zenine, and T. Zenis

\begin{abstract}
The Tile Calorimeter, which constitutes the central section of the ATLAS hadronic calorimeter, is a non-compensating sampling device made of iron and scintillating tiles. The construction phase of the calorimeter is nearly complete, and most of the effort now is directed toward the final assembly and commissioning in the underground experimental hall.

The layout of the calorimeter and the tasks carried out during construction are described, first with a brief reminder of the requirements that drove the calorimeter design. During the last few years a comprehensive test-beam program has been followed in order to establish the calorimeter electromagnetic energy scale, to study its uniformity, and to compare real data to Monte Carlo simulation. The test-beam setup and first results from the data are described. During the test-beam period in 2004, lasting several months, data have been acquired with a complete slice of the central ATLAS calorimeter. The data collected in the test-beam are crucial in order to study algorithms for hadronic energy reconstruction using single particles. The generalization of these algorithms to reconstruct jet energies will be the starting point for numerous physics studies in which jets play a leading role. The results obtained in applying these algorithms to simulated di-jet events are given in the last section of the note.
\end{abstract}

Index Terms-Calorimetry, hadronic, jet, tile, calorimeter.

\section{INTRODUCTION}

$\mathbf{T}$ HE identification and reconstruction of jets play a key role in many of the physics goals of the ATLAS experiment [1], such as the detection of particle resonances decaying into

Manuscript received November 15, 2004; revised October 25, 2005.

Please see the Acknowledgment section of this paper for the author affiliations.

Digital Object Identifier 10.1109/TNS.2006.872628 jets, or in the search for Supersymmetric particles and quark compositness. Moreover, since QCD provides one of the main backgrounds to most new particle searches, efficient jet reconstruction is essential for background rejection.

The requirement of excellent jet identification and reconstruction in the environment of the Large Hadron Collider (LHC) at CERN has guided the design of the ATLAS calorimeter. The hadronic section of the ATLAS calorimeter is comprised of three systems which exploit different measurement techniques as a function of rapidity, in order to optimize the calorimeter performance, while maintaining high resistance to radiation damage. The Tile Calorimeter (TileCal) [2] constitutes the central section in rapidity of the ATLAS calorimeter. The design of the TileCal is discussed in Section I-A, while the construction phase is briefly detailed in Section I-B. The goals and results of the test-beam program are given in the following two sections. The final section is devoted to progress in algorithms of hadronic energy reconstruction.

\section{A. The Tile Calorimeter}

The design of the ATLAS hadronic calorimeters has been driven by the requirement of being hermetic and having a coverage in pseudo-rapidity $(\eta)$ up to \pm 5 , in order to minimize the error in the measurement of missing transverse energy. Moreover, the non-linearity of energy reconstruction in jets up to a few $\mathrm{TeV}$ must be smaller than a few percent and the jet energy resolution must be of the order of $50 \% \sqrt{E(\mathrm{GeV})} \oplus 3 \%$. These requirements have been evaluated from analysis carried out on benchmark physics channels, such as compositness searches, whose signals can be faked by calorimeter non-linearity, and in 
the reconstruction of the $W \rightarrow j j$ mass which demands good hadronic energy resolution. Last, but not least important, are the cell size and the signal integration time, which should be sufficiently small in order to minimize the contribution from pile-up.

TileCal is a sampling device located in the region $|\eta|<1$.7. It is subdivided into a large central barrel and two smaller lateral extended barrels, having a $2.28 \mathrm{~m}$ inner radius and a $4.25 \mathrm{~m}$ outer radius. The entire length of the calorimeter is about $11 \mathrm{~m}$. Each barrel consists of 64 modules, or wedges, of size $\Delta \phi=$ 0.1 , made of iron plates and scintillating tiles. The 4 and $5 \mathrm{~mm}$ thick iron plates are assembled to form a frame with pockets in which $3 \mathrm{~mm}$ thick scintillating tiles are inserted. The assembled module forms an almost-periodic iron-scintillator structure with a ratio by volume of about $4.7: 1$.

The light produced in the scintillating material is collected at two edges of each tile using two wavelength shifting fibers. The fibers are than grouped and coupled to photo-multipliers (Hamamatsu R7877) (PMTs) housed at the outer edge of each module. The fiber grouping allows to define a three-dimensional cell structure in such a way as to form three radial sampling depths $(1.4,4.0$, and $1.8 \lambda$ thick) composed by cells having dimensions equal to $\Delta \eta \times \Delta \phi=0.1 \times 0.1(0.2 \times 0.1$ in the last layer). The fibers coupled to the two edges of each scintillating tiles are coupled to two different PMTs in oder to guarantee a redundant readout for the signal of each cell. This double readout allows for the partial equalization of signals produced by particles entering the scintillating tiles from different impact positions, as well as enhancing the reliability of the TileCal over its expected ten to fifteen years operation, with limited access for maintenance. The photo-multipliers are housed in soft-iron and $\mu$ metal cylinder that shield the residual solenoid and toroid magnetic field which amounts to about 40 Gauss.

A sketch of the optical readout is given in Fig. 1.

The gap region between the barrel and the extended barrel is instrumented with special modules made of iron-scintillator sandwiches, having the same sampling fraction as the rest of the calorimeter, and with thin scintillator counters where the free space is limited. These devices allow to partially recover the energy lost in the crack regions of the detector.

The signal generated by each photo-multiplier is shaped and injected to two amplification circuits whose gains are in the ratio 1:64. The two amplified signals are then sampled and digitized at $40 \mathrm{MHz}$ by two 10-bit Analog to Digital Converters. The full width at half maximum of the shaped signal is about $50 \mathrm{~ns}$, and typically is read out over 9 samples of $25 \mathrm{~ns}$ (Fig. 2). In nominal operation, the digitizer circuit logic selects the signal corresponding to the higher non-saturated gain. The photo-multiplier tubes and all front-end electronics are mounted in an aluminum structure of length $3 \mathrm{~m}$, called a "drawer". Each drawer is inserted in a girder frame along the outer edge of each module. The total number of photo-multipliers in TileCal amounts to about 10000 .

The TileCal is situated just behind the cryostat of the Liquid Argon electromagnetic calorimeter. The total length of the instrumented region at the outer edge of the TileCal is $8.2 \lambda$ (about 80 radiation lengths) including $1.2 \lambda$ of the electromagnetic section. Both the Liquid Argon calorimeter and the TileCal are noncompensating devices, with $e / h$ ratios measured to be about

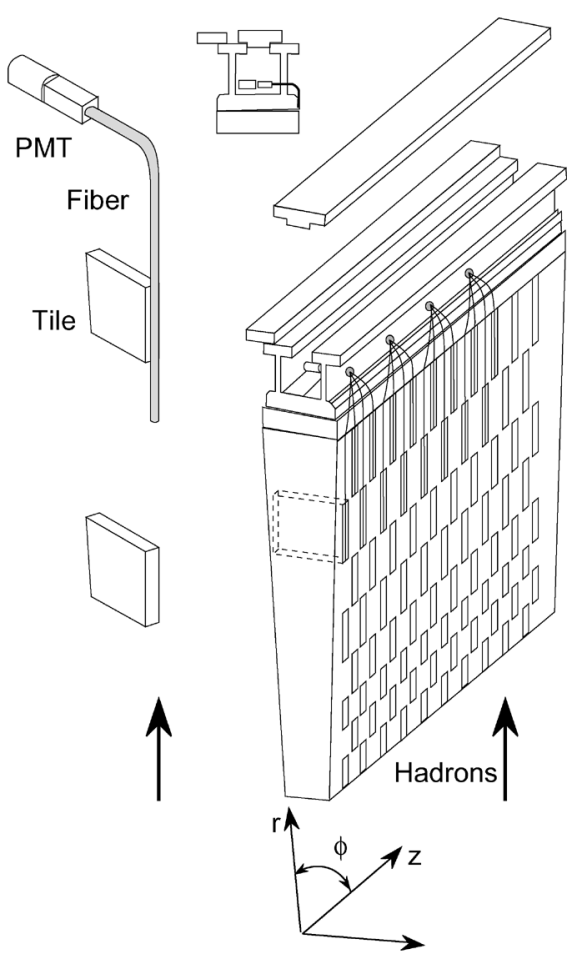

Fig. 1. Sketch of the optical readout of a TileCal module. The various components of the optical readout-tiles, fibers, and the photo-multipliers (PMT) - are shown on figure. Hadrons produced centrally at the interaction point cross the calorimeter in the direction indicated on the figure.

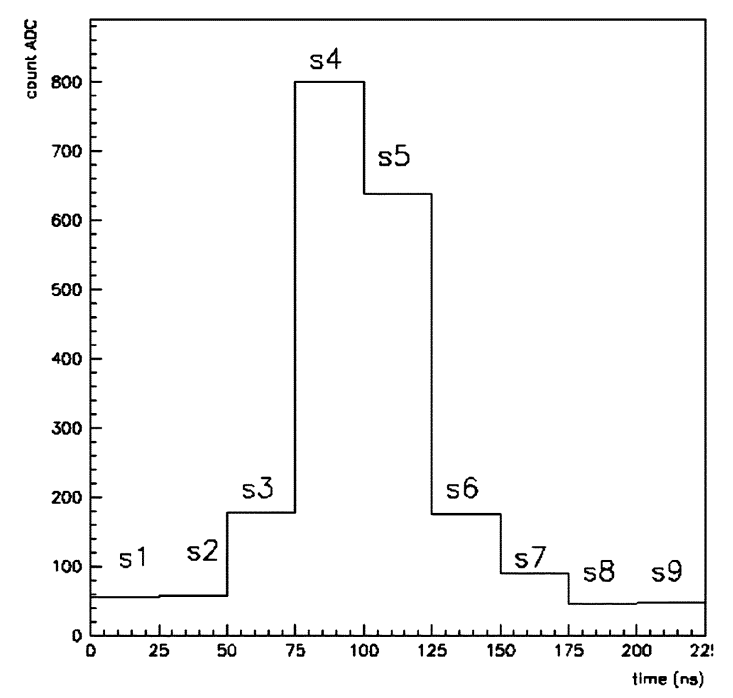

Fig. 2. Example of time pulse shape after the $40 \mathrm{MHz}$ digitization for a calibration signal. The nine time samples are indicated with s1 to s9.

1.5 and 1.4, respectively. Compensation is obtained by reconstruction algorithms, which typically apply different weights to the signal measured in the various longitudinal sampling depths. This procedure allows one to improve both the linearity and the energy resolution of the showers generated by hadronic particles or jets.

The calorimeter is complemented by a triple system of monitoring and/or equalization which allows to test the signals at various stages. The linearity and stability of the electronics and the gains of the two amplification circuits are monitored by 
injecting charge at the input stage of each electronic channel. The readout chain up to the light mixer which acts as the input window for the photo-multiplier is monitored by means of a laser signal. The amplitude of the laser signal covers the whole dynamic range up to the photo-multiplier saturation level. The calorimeter is also equipped with a ${ }^{137} \mathrm{Cs}$ source that can be transported by a hydraulic system to illuminate each individual tile. The signal recorded with this source allows to equalize the response of each cell, by adjusting the voltages of each photo-multiplier. The resulting inter-module equalization obtained with this procedure is within a few per mil. Together, the three control signals described are also used to obtain a finer offline cell inter-calibration.

\section{B. TileCal Construction}

The TileCal project is twelve years old, dating back to the first R\&D program in 1992. Final module production began in 1998. The mechanical assembly of the iron frame, starting from the iron plates, was the responsibility of seven institutes, while the scintillating tiles, made of doped Polystyrene, were produced at a single institute. The production of the 760000 wavelengthshifting fibers was commissioned to the Kuraray Company in Japan. The fibers were delivered in bunches to two institutes, where the quality control procedures were carried out, on a $1 \%$ randomly-selected sample, using dedicated test benches. These tests allowed to accept or reject the fiber bundles in a short time after production.

Further dedicated test benches were built at seven institutes in order to characterize and select the about 10000 photo-multipliers produced by Hamamatsu.

The iron frames, the scintillating tiles and the fibers were then delivered to CERN, where the final opto-mechanical assembly and certification were performed.

The electronic components housed in the drawer of each module are produced by 17 institutes, while their electro-mechanical assembly and certification is centralized at a single institute. Once the drawers are completed and tested, they are delivered to CERN where they are inserted into the modules. A final module certification is carried out using a charge injection system, coupled at the first stage of the electronic read-out, and a movable ${ }^{137} \mathrm{Cs}$ source which allows scan all tiles in the module.

The further components that complete the system are the high and low voltage modules. The high voltage modules, designed for the TileCal project and produced by a commercial company, are placed in an external crate, and cabled via patch panels to the high voltage distributor system on each drawer, allowing for the adjustment of each photo-multiplier voltage. The low voltage system, specifically designed for TileCal, is integrated into the outer edge of each module. The production of these low voltage modules is still ongoing and is foreseen to be completed in 2005.

In order to check the geometrical tolerance (a few millimeters over a few meters) and the complicated integration with cables, services and other detectors, the three TileCal barrels have been pre-assembled on the surface. The first calorimeter barrel completely pre-assembled is shown in Fig. 3. All the problems encountered in completing this task provided very useful experience for the later assembly in the pit. The last extended-barrel

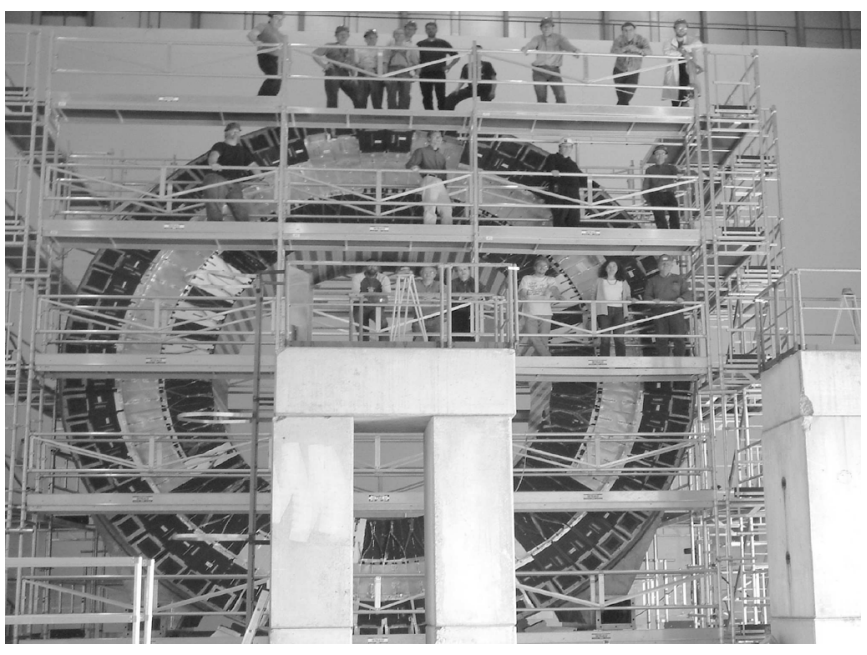

Fig. 3. The first completely pre-assembled extended barrel of the Tile Calorimeter placed behind the scaffolding.

pre-assembly was completed in September 2004, while the assembly of the first half of the barrel was completed in the experimental hall in April 2004. The three barrels of the TileCal should all be assembled in the underground experimental area by December 2005 .

\section{Test-Beam Programs and Selected Results}

During the last five years, the main effort beside construction has been dedicated to carry on the test-beam program. The primary aim of the test-beam has been to calibrate the electromagnetic energy scale of the calorimeter. This has been accomplished during numerous data-taking periods, each lasting a few weeks, between the years 2000 and 2003 .

During 2004, a combined test-beam period spanning several months has allowed to collect the first data using a vertical slice of ATLAS. During this period, for the first time, an entire section of the ATLAS calorimeter, both electromagnetic and hadronic, has collected common data using calorimeter modules with the final ATLAS configuration. In the next two subsections, the aims, the detector setup, and selected results of the test-beams are reviewed.

1) The Standalone Test-Beam: Fig. 4 shows a picture of the setup used during the tests carried out at the $\mathrm{H} 8$ beam-line of the CERN SPS accelerator. In each data-taking period, four calorimeter modules were placed on a movable table and exposed to beam. The bottom module on the table is the prototype barrel module (referred to as "Module 0"), which has been used as a reference during all data-taking periods. Stacked on the top of "Module 0" are one barrel module and two extended barrel modules placed next to one another. This configuration allows for a complete containment of the hadronic shower produced by pion interactions in the central barrel module. The movable table (visible in Fig. 4) allows to direct the beam on a module at any point along the lateral side (on Fig. 4, the beam enters the modules from the left side). The setup also allows one to point the beam along the ATLAS detector inner edge of the module, as is the case for particles produced by beam interactions in ATLAS. During all the test-beam periods about $12 \%$ of the calorimeter modules were exposed to beam. 


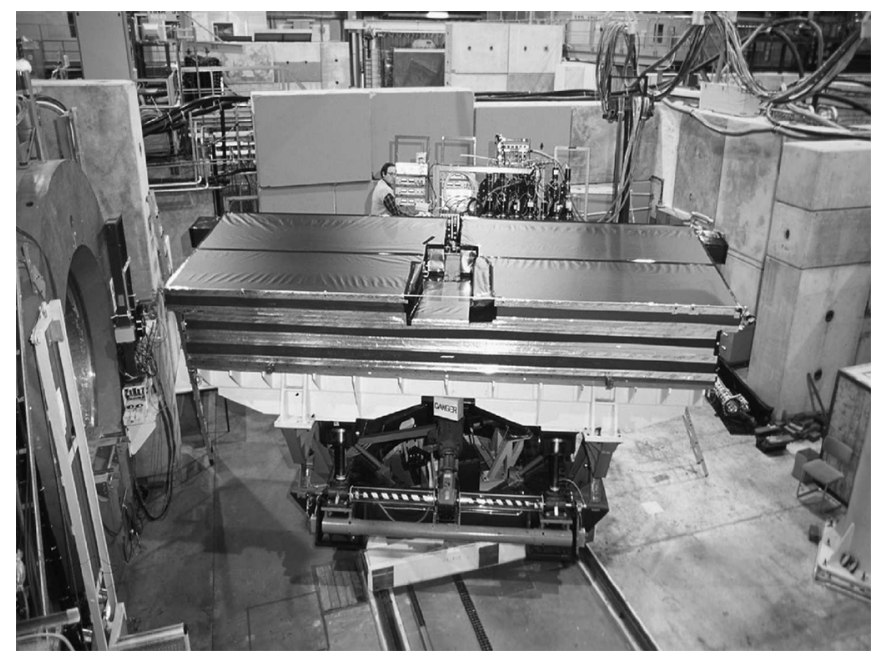

Fig. 4. Picture of the setup of the TileCal modules at the standalone test-beam. The beam enters the modules from the left side and impinges on the modules stacked on the movable table. The table allows to rotate, shift and lift the modules with respect to the beam direction.

During initial setup before each test-beam, the first step in the calibration procedure consists of the equalization of the module response using the Cesium source. The effectiveness of the equalization procedure is evaluated by the residual Cesium signal dispersion after the equalization procedure has been applied. A typical distribution of the Cesium signal recorded in each cell of a half barrel module is shown the top plot of Fig. 5 . The equalization obtained with this procedure is of the order of a few per mill as it is shown in the bottom plot of Fig. 5 where the histogram of the Cesium signal of two complete modules under test is shown.The histogram has a mean value equal to 1741 and a root mean square equal to 3.116 that is about $0.18 \%$. The residual signal dispersion are due to differences among the tiles, iron plates and fibers belonging to the same cell.

During the equalization procedure, the photo-multiplier gains are adjusted to measure about $1.2 \mathrm{pC} / \mathrm{GeV}$ for electrons incident on the inner longitudinal cells of the calorimeter. The precise value of the electromagnetic calibration factor obtained with this procedure is then measured using electron beams of energies ranging from 20 to $180 \mathrm{GeV}$. The movable table allows one to measure this calibration factor over all the cells, with the beam entering from different angles. The distribution of the calibration factors obtained for various cells, configurations, energies and different modules is plotted in Fig. 6. This distribution gives a mean calibration factor equal to $1.21 \mathrm{pC} / \mathrm{GeV}$ and a root mean square equal to 0.04 , resulting in a cell-to-cell uniformity of the electromagnetic scale better than $3.5 \%$. In fact, this should only be considered as an upper limit, since finer offline adjustments are still to be applied, depending on the details of the signal reconstruction algorithm used. The equalization for the inner cells are checked using muon beams. These studies also show that the uniformity is within a few percent.

A large data sample of pion beams has been acquired using various configurations and energies ranging from 20 to 180 $\mathrm{GeV}$. These data are used to tune the Geant4 [3] detector simulation. The comparison of test-beam data with Geant 4 simulated data [4][5] shows that while the description of the
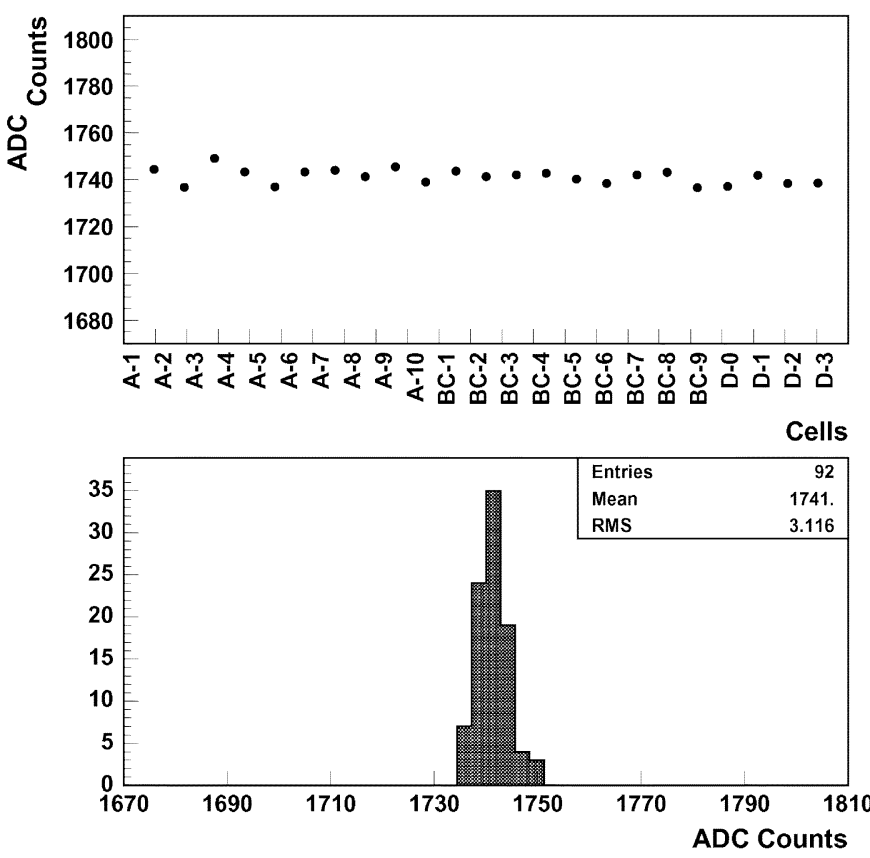

Fig. 5. Distribution of the Cesium signal as a function of the cell number (top) recorded for each cell of half barrel module after the equalization procedure has been applied. Each cell is labeled by a letter and number and the minus sign indicate the negative pseudo-rapidity region. Cells A belong to the first longitudinal sample while cells BC and D to the second and third ones respectively. The histogram of the cell signals for two complete modules under test (Module 0 is not considered), after the equalization, is shown on the bottom plot. The residual signal dispersion is about $0.2 \%$.

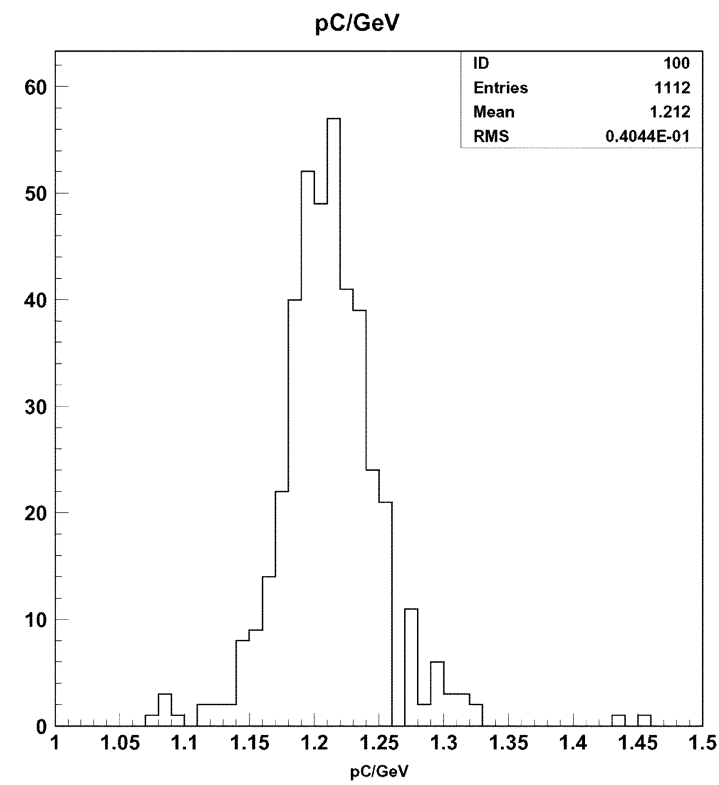

Fig. 6. Distribution of the electromagnetic calibration factor, in $\mathrm{pC} / \mathrm{GeV}$, obtained for different cells, modules, geometries and energies. The number of entries in the histogram is about 400 , the mean is equal to $1.21 \mathrm{pC} / \mathrm{GeV}$ and the root mean square is $3.3 \%$.

resolution and $e / \pi$ ratio as a function of the energy are modeled within a few percent, the longitudinal and transverse shower profiles are not yet reproduced at a satisfactory level and require more studies and tuning.

A special data-taking period has been devoted to very low energy ( 1 to $10 \mathrm{GeV}$ ) beams. The high multiplicity of low energy 


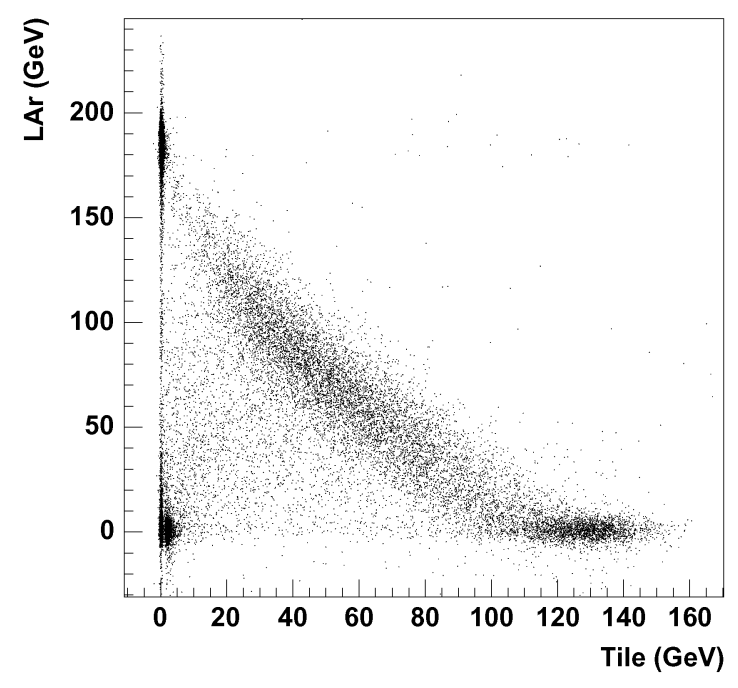

Fig. 7. Distribution of the correlation between the signal recorded in the electromagnetic section (LAr) and hadronic section (Tile) of the calorimeter at the combined test-beam for a mixed pion/muon/electron beam of energy $180 \mathrm{GeV}$. The energy scales are roughly calibrated to the electromagnetic scale.

tracks found in jets makes this data very important to model the detector response to jets.

2) The Combined Test-Beam: The last test-beam period before the start of ATLAS has been carried out from May to November 2004 on a complete vertical slice of the central ATLAS detector. Most of the devices tested were in their final configuration and equipped with the final front-end electronics.

The movable table used for the standalone test-beams has been modified to mount, in front of the TileCal modules, a cryostat containing three modules of the electromagnetic Liquid Argon calorimeter. The amount of dead material lying between the electromagnetic calorimeter and TileCal is slightly smaller than that expected in ATLAS. In order to select events in which the primary shower began in the TileCal modules, a scintillation counter has been placed just downstream of the cryostat. A second scintillation counter has been placed further downstream of the TileCal modules in order to select muons. On the scatter plots in Fig. 7 one sees the correlation between the signals recorded in the electromagnetic (LAr) and the hadronic calorimeter sections (Tile) for a mixed pion/muon/electron beam of energy $180 \mathrm{GeV}$. The correlation plot regions populated by muons (bottom left), electrons (top left) and pions (bottom right) are easily identifiable. Three extended barrel modules were placed on a second movable table. This setup allows for the beam to be aimed at the calorimeter with a direction corresponding to a pseudo-rapidity ranging from 0.2 to 1.2. The beam energy was varied from 1 up to $350 \mathrm{GeV}$. Thus, with the data acquired during these tests, a complete study of the characteristics of hadron energy reconstruction, including effects due to the crack region placed between the barrel and extended barrel, will be possible.

The final data have been recorded in October 2004, so the analysis of pion data has just begun and the first step will be to set the correct electromagnetic scale for both calorimeter sections. Then, the algorithms to reconstruct the hadronic energy, compensating for the $e / h>1$, may be tested. In the next section the performance of one of these algorithms on simulated single particles and jets is discussed.

\section{Hadronic Energy Reconstruction}

The aim of the reconstruction process is to identify jets from calorimetric clusters to relate their kinematic properties to those of the parent partons. In order to reconstruct the hadronic energy from the calorimeter signal, a calibration procedure has to be applied to take into account both detector effects (e/h and dead material) and physics effects (energy lost in the jet reconstruction process, underlying event, pile-up, initial and fine state radiation). ATLAS approach is to apply these calibration in two separate steps, the first one takes care of detector effects only and the second one correct for physics related effects.

The most commonly used method to implement the first stage is inspired to the technique used by the $\mathrm{H} 1$ collaboration [6]. The basic idea of this method is to correct upward the response of cells with relatively low signal-typically hadronic - in order to equalize their response to that of cells with large deposited energy - typically electromagnetic. Clearly the method works better if the granularity of the calorimeter is well dimensioned with respect to electromagnetic deposit.

The total reconstructed energy $\left(E_{\mathrm{rec}}\right)$ is obtained as a linear weighted sum of the energy deposited in the cells $\left(E^{\text {cell }}\right)$ of each longitudinal calorimeter sample:

$$
E_{\mathrm{rec}}=\sum_{i, j} w_{i}\left(E^{\mathrm{cell}}, E_{\mathrm{MC}}\right) E_{i, j}^{\mathrm{cell}}
$$

where $i$ runs over all the longitudinal samples and $j$ over all the cells of the $i$-th sample. The weights $w_{i}$ depends on the cell energy deposits and on the total energy of the ensemble of particles belonging to the jet (MC jet), indicated here as Monte-Carlo energy $E_{\mathrm{MC}}$. The weights are obtained by minimizing the energy resolution with respect to the true total energy, applying the constraint that the total reconstructed energy after re-weighting is equal to the true energy. Since in real data the value of the true energy is not available, an iterative procedure is applied. An initial estimate of the energy is obtained using a simple method, such as the unweighted sum of the cell energy, and this is used for the initial estimate of the weights. Then the calibrated energy thus obtained is used as an input to calculate new weights and a new estimate of the energy. After a few iterations of this procedure, the reconstructed energy does not vary by more than few percent [7].

This method has been already used both on real and simulated data, and it is able to restore the linearity to within a few percent, and to improve the energy resolution [1][8]. Recently this procedure has been implemented in the new ATLAS software and has been applied to samples of QCD di-jet events generated with PYTHIA6.3. The detector simulation was based on Geant3 [9]. The results obtained on linearity and energy resolution for jets in the central $(\eta<0.7)$ ATLAS region are shown in Fig. 8. Non-linearities after calibration are within $2 \%$ and a significant improvement in energy resolution is obtained. The same procedure has been applied to single pion events generated with 


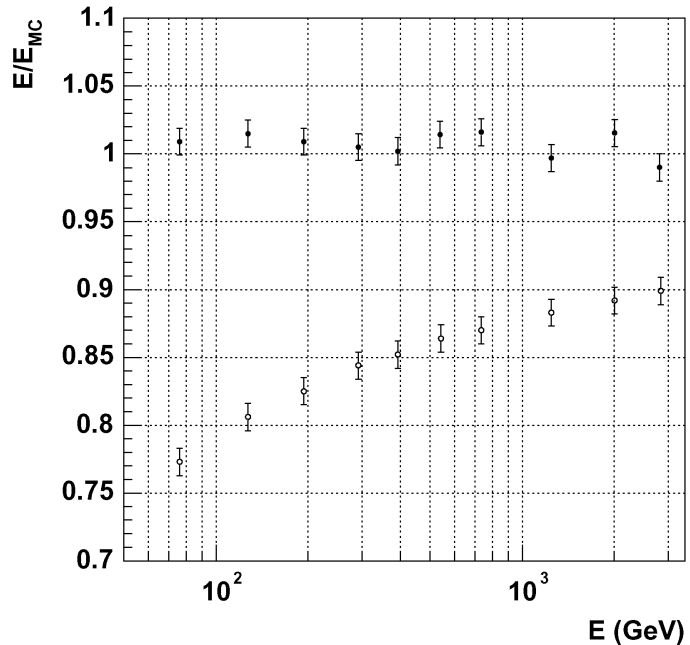

(a)

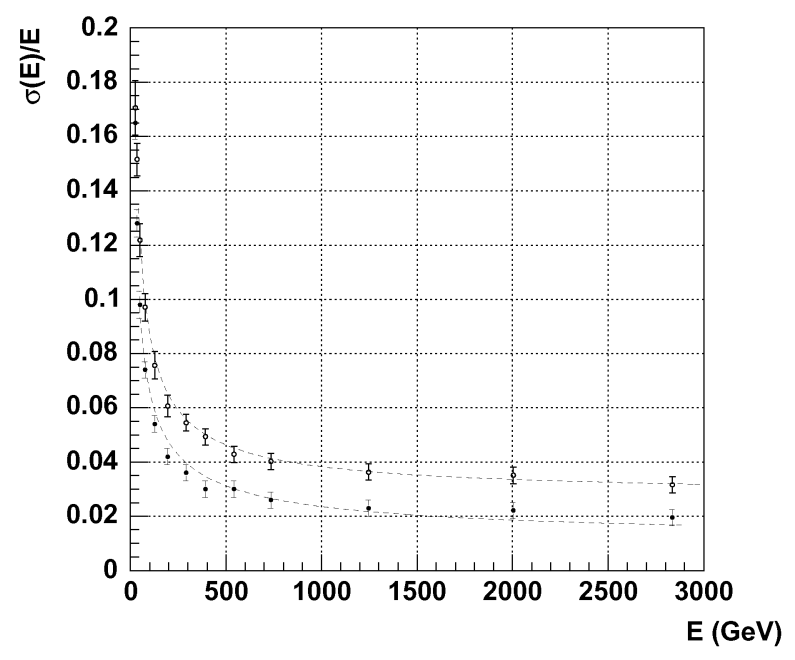

(b)

Fig. 8. Energy linearity and resolution as a function of the Monte Carlo jet energy, obtained for a sample of QCD di-jet events where the detector is simulated using Geant3 (di-jet events simulated using Geant4 package are not yet available). Electronics noise and pile-up have not been considered. Events in the pseudo-rapidity region $|\eta|<0.7$ have been selected. In each plot the results before (open circle) and after (solid circle) the calibration procedure has been applied are shown. (a) Jet energy linearity, (b) Jet energy resolution.

the Geant 4 detector simulation. The linearity after calibration is well within $1 \%$ and the energy resolution is in agreement with the design resolution [2].

The second stage of calibration allows to reconstruct the parton kinematics imposing the transverse momentum balance in events where the jet is reconstructed back-to-back to well measured particles. A standard calibration sample consists of $Z+$ jets event, with $Z \rightarrow \mu^{+} \mu^{-}$or $Z \rightarrow e^{+} e^{-}$. These calibration samples allows as well to calibrate b-jets in fact using standard selection criteria the $Z+$ jet sample contains about $5 \%$ of $Z+\mathrm{b}$. Statistical errors on the jet scale of the order of $1 \%$ can be reached in about six months for $50 \mathrm{GeV}<p_{T}<400$ $\mathrm{GeV}$ [10]. Other calibration samples consists of $\gamma+$ jets or of $W \rightarrow$ jetjet where the $\mathrm{W}$ mass is use to set the calibration scale. The refined strategy to determine the parton energy scale is under study and it will be precisely defined using real data.

\section{CONCLUSION}

The opto-mechanical assembly of modules of the TileCal was completed at the beginning of 2004. By April 2004, the first half of the barrel was assembled in the experimental pit. The installation of the front-end electronics is nearly complete, and the last stage, the installation of the low voltage power supplies, will begin in 2005. During the same period, the modules already assembled in the experimental pit will be tested and the first data-taking with the final system using cosmic ray muons will begin. Parallel to the assembly in the pit, offline software development will continue, including the study of algorithms for jet energy reconstruction. Particularly important for these studies are the data recently acquired in the combined test-beam.

The TileCal construction phase is nearly finished, the assembly and commissioning in the pit is proceeding, and preparations are underway to analyze first collision data in 2007.

\section{ACKNOWLEDGMENT}

This paper is the result of a collective effort that is due also to people not belonging to the TileCal group. The authors would like to thank all the ATLAS Trigger and Data Acquisition group and the ATLAS Liquid Argon Electromagnetic Calorimeter group. They are in particular grateful to all the technicians that helped with the calorimeter assembly and to the Test Beam Coordinator B. Di Girolamo. Finally, the authors would like to thank the SPS staff that allowed them to collect the test-beam data.

P. Adragna, V. Cavasinni, D. Costanzo, T. Del Prete, A. Dotti, V. Flaminio, A. Lupi, E. Mazzoni, C. Roda, F. Sarri, G. Usai, and I. Vivarelli are with Pisa University and INFN, Pisa, Italy.

C. Alexa, V. Boldea, S. Constantinescu, S. Dita, and D. Pantea are with National Institute for Physics and Nuclear Engineering, Bucharest, Romania.

K. Anderson, A. Farbin, A. Gupta, M. Hurwitz, F. Merritt, M. Oreglia, J. Pilcher, H. Sanders, M. Shochet, F. Tang, and R. Teuscher are with University of Chicago, Chicago, Illinois, USA.

A. Antonaki, D. Fassouliotis, V. Giakoumopoulou, N. Giokaris, M. Lembesi, and A. Manousakis are with University of Athens, Athens, Greece.

V. Batusov, J. Budagov, M. Liablin, Y. Lomakin (October 2003), S. Maliukov, I. Minashvili, V. Romanov, N. Russakovich, A. Sissakian, N. Topilin, and V. Vinogradov are with JINR, Dubna, Russia.

P. Bednar, I. Fedorko, I. Sykora, S. Tokar, and T. Zenis are with Comenius University, Bratislava, Slovakia.

C. Biscarat, D. Calvet, C. Ferdi, V. Garde, P. Gris, C. Guicheney, R. Lefevre, G. Montarou, D. Pallin, F. Podlyski, P. Rosnet, P. Roy, C. Santoni, L.-P. Says, and F. Vazeille are with LPC Clermont-Ferrand, Université Blaise Pascal/CNRS-IN2P3, Clermont-Ferrand, France.

G. Blanchot, M. Bosman, M. Cavalli-Sforza, I. Korolkov, L. Miralles, O. Norniella, X. Portell, O. Saltó, M. Volpi are with Institut de Fisica d'Altes Energies, Universitat Autònoma de Barcelona, Barcelona, Spain.

A. Bogush, V. Gilewsky, Yu. Kurochkin, and I. Satsunkevitch are with Institute of Physics, National Academy of Sciences, Minsk, Belarus.

C. Bohm, S. Holmgren, K. Jon-And, J. Klereborn, M. Ramstedt, and B. Selldèn are with Stockholm University, Stockholm, Sweden.

C. Bromberg, J. Huston, R. Miller, and R. Richards are with Michigan State University, East Lansing, Michigan, USA.

L. Caloba, A. S. Cerqueira, D. O. Damazio, C. Maidantchik, F. Marroquim, J. M. Seixas, and P. Da Silva are with COPPE/POLI/UFRJ, Rio de Janeiro, Brazil. 
J. Carvalho and J. Pinhão are with LIP and FCTUC University of Coimbra, Portugal.

J. Castelo, M. V. Castillo, C. Cuenca, A. Ferrer, E. Fullana, V. Gonzalez, E. Higon, C. Iglesias, J. M. Lopez Amengual, J. Poveda, B. Salvachua, E. Sanchis, J. Torres, and J. A. Valls are with IFIC, Centro Mixto Universidad de ValenciaCSIC, E46100 Burjassot, Valencia, Spain.

M. Cobal, B. Di Girolamo, I. Efthymiopoulos, O. Gildemeister, P. Grenier, A. Henriques, F. Martin, M. Nessi, G. Schlager, and F. Spanó are with CERN, Geneva, Switzerland.

F. Cogswell, R. Downing, D. Errede, S. Errede, M. Haney, T. Junk, V. Simaitis, and I. Vichou are with University of Illinois, Urbana-Champaign, Illinois, USA

M. David, A. Gomes, A. Maio, C. Marques, J. Pina, J. G. Saraiva, J. Silva, and J. Santos are with LIP and FCUL University of Lisbon, Portugal.

T. Davidek, J. Dolejsi, Z. Dolezal, P. Krivkova, R. Leitner, M. Suk, P. Tas, and S. Valkar are with Charles University in Prague, Prague, Czech Republic.

K. De, J. Li, M. Sosebee, A. Vartapetian, and A. White are with University of Texas at Arlington, Arlington, Texas, USA.

A. Fenyuk, A. Karyukhin, A. Miagkov, A. Solodkov, O. Solovianov, E. Starchenko, A. Zaitsev, and A. Zenine are with Institute for High Energy Physics, Protvino, Russia.

V. Guarino, T. Le Compte, L. Nodulman, L. E. Price, J. Proudfoot, J. Schlereth, R. Stanek, and D. Underwood are with Argonne National Laboratory, Argonne, Illinois, USA.

H. Hakobyan and M. Simonyan are with Yerevan Physics Institute, Yerevan, Armenia.

J. Khubua is with JINR, Dubna, Russia, and also with HEPI, Tbilisi State University, Tbilisi, Georgia.

Y. Kulchitsky is with JINR, Dubna, Russia and Institute of Physics, National Academy of Sciences, Minsk, Belarus

P. Kuzhir, V. Rumiantsau (february 2003), P. Shevtsov, and P. Starovoitov are with National Centre of Particles and High Energy Physics, Minsk, Belarus.
M. Lokajicek, S. Nemecek, and L. Pribyl are with Institute of Physics, Academy of Sciences of the Czech Republic, Prague, Czech Republic.

A. Onofre is with LIP and University Católica Figueira da Foz, Portugal.

\section{REFERENCES}

[1] ATLAS Physics Technical Design Report. ATLAS Collaboration, CERN/LHCC/99-15.

[2] Tile Calorimeter Technical Design Report. ATLAS Collaboration, CERN/LHCC/96 42.

[3] S. Agostinelli, "GEANT4: A simulation toolkit," Nucl. Instr. Meth. A, vol. 506, pp. 250-303, 2003.

[4] C. Alexa, Geant4 Hadronic Physics Validation With LHC Test-Beam Data: First Conclusions. CERN-LCGAPP-2004-10 [Online]. Available: http://lcgapp.cern.ch/project/docs/hadronic-note.ps

[5] A. Dotti, A. Lupi, and C. Roda, "Results from ATLAS Tile Calorimeter: a comparison between data and Geant 4 simulation," Proc. 9th Topical Seminar on Innovative Particle and Radiation Detectors [Online]. Available: http://www.bo.infn.it/sminiato/sm04/paper/dottiSiena04.ps.gz., to be published in Nucl. Phys. B.

[6] "H1 Collaboration," Nucl. Instrum. Methods, vol. A386, p. 348, 1997.

[7] M. P. Casado and M. Cavalli Sforza, H1-inspired analysis of the 1994 combined test of the Liquid Argon and Tilecal calorimeter prototypes. ATLAS-TILECAL-96-075, Apr. 1996.

[8] "ATLAS Collaboration," Nucl. Instrum. Methods, vol. A449, pp. 461-477, 2000.

[9] Geant, Detector Description and Simulation Tool. CERN Program Library Writeup W5013. Application Software Group Computing and Network Division, CERN.

[10] R. Lefevre and C. Santoni, In situ determination of the scale and resolution of the jet energy measurements using $\mathrm{Z} 0+$ jet events. ATL-PHYS2002-026. 